\title{
Análisis de la relación entre ansiedad rasgo y experiencia profesional en la danza
}

\author{
Paula De Castro Fernández \\ CSDMA y Universidad Rey Juan Carlos (España)
}

Este estudio investiga la relación entre los niveles de AR de los bailarines profesionales y su grado de experiencia en el mundo de la danza. Para ello se ha trabajado con bailarines de tres compañías de danza internacionales: la Compañía Nacional de Danza en España (CND), el Ballet Nice Méditerranée de la Ópera de Niza en Francia (BON) y el Béjart Ballet Lausanne en Suiza (BBL). Se aplicó el STAI de Spielberger a 44 bailarines con el objetivo de determinar su nivel de ansiedad rasgo y las posibles correlaciones entre las variables AR y años de experiencia profesional. Los resultados muestran que un 56.8\% de los sujetos presentan una AR situada en el percentil 10. La media de la variable relativa a la experiencia como profesionales del sector de la danza de los sujetos es de 9.34 años, y los resultados del análisis de la correlación entre AR y experiencia profesional se revelan no significativos. Podemos concluir que el bailarín consagrado presenta unos muy bajos niveles de AR que no se relacionarían de forma significativa con una mayor o menor experiencia en el ámbito profesional. La AR parece ser un rasgo de gran estabilidad, o bien realmente característico del bailarín profesional, o no influenciable por su grado de experiencia escénica.

Palabras clave: Ansiedad, bailarín, danza, experiencia, profesional.

Analysis of the relationship between trait anxiety and professional experience in dance. This study investigates the relationship between the TA levels of professional dancers and their level of experience in the world of dance. We have worked with dancers from three international dance companies: the National Dance Company in Spain (CND), the Ballet Nice Méditerranée of the Opéra de Nice in France (BON) and the Béjart Ballet Lausanne in Switzerland (BBL). The STAI from Spielberger was applied to 44 dancers in order to determine their level of anxiety trait and the possible correlations between the TA variables and years of professional experience. The results show that $56.8 \%$ of the subjects presented a TA located in the 10 percentile. The average of the variable related to the experience as professionals in the dance sector of the subjects was 9.34 years, and the results of the analysis of the correlation between TA and professional experience are not significant. We can conclude that the consecrated dancer presents very low levels of TA that would not be significantly related to a greater or lesser experience in the professional field. The TA seems to be a feature of great stability, either really characteristic of the professional dancers, or not influenced by their degree of stage experience.

Keywords: Anxiety, dancer, dance, experience, professional.

Correspondencia: Paula De Castro Fernández. CSDMA y Universidad Rey Juan Carlos. Ctra. de Húmera, $\mathrm{n}^{\circ}$ 87, p3, $2^{\circ} \mathrm{C}$. C.P.: 28224. Pozuelo de Alarcón, Madrid (España). E-mail: pdcf1972@hotmail.com 
La ansiedad es uno de las patologías que más afectan a los profesionales de la danza. Tal y como explica Martínez-Otero (2003), la ansiedad puede resultar beneficiosa si actúa en su justa medida y como factor de adaptación, ya que, al igual que el estrés, incide en la activación del organismo y facilita su rendimiento. No obstante, el estrés excesivo o disestrés y la ansiedad considerada patológica sí interfieren en la homeostasis del organismo, con consecuencias nocivas tanto para la salud como para el rendimiento de los profesionales de la danza.

Un aspecto diferenciador entre el estrés y la ansiedad, es la relevancia que tiene en el primero el componente fisiológico, ya que en la ansiedad, así como en el miedo, pese a poseer un claro componente fisiológico, el psicológico resulta crucial. Tanto el estrés como el miedo poseen un carácter adaptativo, y son respuestas orgánicas normales cuya función es la de hacer frente a las demandas externas o estresores. Solamente podemos considerar la posibilidad de que el estrés se torne en patología si las respuestas adaptativas del sujeto se prolongan excesivamente en el tiempo y no consiguen hacer frente a los estresores. Por tanto, y contrariamente a la ansiedad, el estrés y el miedo no se consideran condiciones patológicas.

Si un rasgo de personalidad es una disposición relativamente permanente, el estado de ánimo es una respuesta psicológica bastante efímera a un estímulo externo, que interviene en una situación dada.

La ansiedad es un factor que depende tanto del ambiente laboral que rodea al profesional, como de sus rasgos de personalidad. Por este motivo resulta necesario estudiar con profundidad este aspecto, con el objetivo de analizar y comprender las características del artista dedicado profesionalmente a la danza, y poder entonces actuar implementando estrategias concretas que ayuden a paliar las problemáticas propias de enfrentamiento diario al trabajo.

Se han realizado estudios sobre la relación entre la personalidad del deportista y el nivel de su "performance" deportiva. Algunas de las conclusiones de esos estudios resultan inesperadas y altamente interesantes: "Numerosos rasgos de personalidad oponen a deportistas y no deportistas"; "Las personas con ciertos rasgos de personalidad se sienten atraídas por el deporte"; "Según el tipo de deporte, los atletas presentan perfiles de personalidad diferentes"; "En algunos deportes, existe una relación entre la personalidad del jugador y el puesto que ocupa en el campo"; "Es posible distinguir atletas de alto nivel de los de nivel inferior basándose en criterios de personalidad"; "Las atletas femeninas se asemejan mucho a los atletas masculinos en términos de personalidad", etc.

Si un rasgo de personalidad es una disposición relativamente permanente, el estado de ánimo es una respuesta psicológica bastante efímera a un estímulo externo, que interviene en una situación dada. En el caso de los deportistas, se suelen estudiar los diferentes estados de ánimo como: tensión/ansiedad, depresión/melancolía, odio/hostilidad/angustia, vigor/actividad, fatiga/inercia y confusión/desconcierto 
(observados con el test POMS, por ejemplo). El perfil de estado de ánimo del deportista de élite es denominado perfil de iceberg, porque todos los estados negativos se sitúan bajo la media de la población estándar, mientras que el vigor se encuentra por encima. A través de los diversos modelos teóricos que se han elaborado, se ha demostrado la influencia del estado de ánimo sobre el comportamiento del deportista. La depresión aparece como una variable moderadora que condiciona la influencia de los otros estados de ánimo sobre el resultado deportivo. Parece que la depresión amplifica los efectos negativos del estado anímico sobre dichos resultados.

Uno de los factores estudiados por la Psicología del deporte es la influencia que los altos niveles de ansiedad del deportista ejercen sobre su rendimiento. Con el fin de optimizarlo al máximo, es necesario aprender a controlar el estrés y la ansiedad durante los entrenamientos y, sobre todo, durante la competición. La denominada "ansiedad situacional precompetitiva" es la ansiedad que aparece frente a una situación de competición. Sus principales antecedentes son el miedo al fracaso y el miedo al juicio social negativo. Pero también existen otras causas: el miedo a lesionarse, la ambigüedad, la perturbación de una rutina, la dificultad de la tarea y diversas variables de personalidad. Todo comportamiento que ayude a un individuo a controlar una situación estresante se considera un comportamiento de "coping".

Como sabemos, el bailarín profesional está equiparado al deportista de alto rendimiento, por lo que podemos aplicar la mayoría de los principios e investigaciones que se realizan desde la Psicología del deporte a la danza profesional.

Según Leal (1997) un atleta de alto rendimiento es un "atleta dotado de condiciones físicas especiales, adecuadas a las exigencias de la disciplina; inmerso en un aprendizaje progresivo de la técnica deportiva, con una energía competitiva para la confrontación y una sustanciosa motivación".

En el caso concreto de los profesionales de la danza, el bailarín es un individuo que vive en constante contradicción física y mental, en continua búsqueda de un equilibrio emocional que se le exige intacto por un lado (la dura disciplina física, que sobrepasa incluso la de la mayoría de los deportes de élite, un enorme autocontrol, un contexto de trabajo basado en jerarquías arcaizantes y conductas despóticas, etc.), al tiempo que se le exige una hipersensibilidad artística y un vaivén emocional extremo que colme la demanda expresiva. Si además añadimos la enorme presión psicológica, tanto la inherente a la persona (ambición personal, miedo a la auto decepción, a las lesiones) como la externa (exigencias de los superiores, alta y constante competitividad, miedo al fracaso, miedo a las críticas, a la pérdida del "estatus"...), resulta obvio que la danza posee todas las características del deporte de élite sumadas a las de una exigente disciplina artística.

Para Garfield (1987): "la mayoría de los atletas reconocen que del 60 al 90 por ciento del éxito en el deporte se debe a factores mentales y al dominio psicológico”, y en la experiencia personal de muchos deportistas de élite se afirma que la planificación del 
entrenamiento psicológico con los componentes físicos, técnicos y tácticos supone el 50\% de la efectividad en las potencialidades del atleta.

El ejercicio promueve una reducción significativa de la AE y las medidas fisiológicas asociadas. De acuerdo con el meta-análisis de Petruzzello et al. (1991), examinando la AE, la AR y los correlatos fisiológicos de la ansiedad, el ejercicio está claramente asociado a una reducción de esas tres medidas, según el sexo, la edad, y el modelo de salud mental (Folkins y Sime, 1981; Morgan, 1973, 1997).

Por ende, en el deporte de élite, el individuo con una AR elevada es generalmente más propenso a experimentar sentimientos de $\mathrm{AE}$ más elevados que la persona con nivel bajo de AR.

Según los trabajos de Spielberger (1966) y Spielberger et al. (1971) los bailarines con mayor AR presentarán mayor AE que otros con una AR más baja, como consecuencia de que los bailarines con una mayor AR suelen percibir las situaciones como más amenazadoras. Deducimos, entonces, que los bailarines con mayor AR son más propensos a perder el control de la calma en momentos clave, y a elevar sus niveles de AE ante una actuación.

Scalan y Passner (1977) indican igualmente que la AR correlaciona significativamente con la $\mathrm{AE}$ que los artistas experimentan momentos antes de la actuación. De igual modo, al finalizar ésta, la AE correlaciona significativamente con el grado de éxito o fracaso obtenido, por lo que los bailarines con menor éxito muestran una mayor ansiedad.

Otro aspecto relevante es que la ansiedad pre-actuación sigue el modelo de la U invertida. Se trata de un modelo propuesto originalmente por Yerkes y Dodson (1908), basado en que la ejecución se ve facilitada por el aumento de la activación (nivel de arousal) hasta alcanzar un punto óptimo a partir del cual, los incrementos resultan en detrimento de la ejecución. Fenz (1975), Fenz y Jones (1972), Lowe (1973) y Gould et al. (1983), han realizado estudios con deportistas de diferentes modalidades que corroboran el modelo de la $\mathrm{U}$ invertida, y evidenciando que niveles moderados de arousal permiten mejores ejecuciones que los niveles extremadamente altos.

La elevada ansiedad antes y durante la actuación que experimenta un bailarín profesional conlleva un alto nivel de arousal, así como sentimientos de tensión y miedo; elementos que describen la ansiedad tal y como Levitt (1980) la comprende, es decir, como un sentimiento subjetivo de temor, acompañado de un elevado nivel de arousal fisiológico.

Por otra parte, surge el interrogante en torno a la relación entre la experiencia escénica de los bailarines y la variabilidad de sus valores de AR. Podemos suponer que a mayor experiencia escénica, el bailarín logrará gestionar la potencial situación de estrés de modo que sus niveles de ansiedad no se vean afectados, y con ello, tampoco su rendimiento. Sin embargo, es posible que la AR del individuo juegue un papel 
preponderante en este tipo de situaciones, y que pese a los años de experiencia profesional, el bailarín mantenga su nivel de activación y de respuesta ansiosa ante el espectáculo.

En base a estudios anteriores sobre los niveles de ansiedad en la danza profesional (De Castro, 2011), intentaremos avanzar en la definición de la personalidad del bailarín profesional, en este caso centrándonos en los aspectos citados anteriormente.

\section{MÉTODO}

\section{Participantes}

Se ha trabajado con 44 bailarines (23 mujeres y 21 hombres) de tres compañías de danza internacionalmente reconocidas: la Compañía Nacional de Danza en España (CND), el Ballet Nice Méditerranée de la Ópera de Niza en Francia (BON) y el Béjart Ballet Lausanne en Suiza (BBL). La edad media general es de 27 años, 31 en la CND, 26 en el BON y 24 en el BBL.

\section{Instrumentos}

Se aplicó el Cuestionario de Ansiedad Estado-Rasgo (STAI) de C.D. Spielberger $(1966,1983)$ en sus versiones inglesa, española y francesa, con el objetivo de determinar los niveles de AR de la muestra.

El test STAI de C.D. Spielberger presenta coeficientes de solidez psicométrica entre 0.86 y 0.95 . El coeficiente de replicabilidad test-retest se encuentra entre 0.65 y 0.89 y muestra considerables evidencias sobre la validez de constructo, fiabilidad y confiabilidad (Spielberger et al., 1983).

\section{Procedimiento}

El test STAI ha sido aplicado a los bailarines profesionales seleccionados, tras explicarles el objeto y método de la investigación. Los sujetos han respondido de forma autónoma y una vez recogidas las pruebas se ha procedido a su valoración e interpretación, obteniendo la medida de ansiedad rasgo correspondiente a cada bailarín participante.

\section{Análisis de datos}

Los datos fueron analizaron utilizando el paquete estadístico IBM SPSS Statistics 2.0. Se trabajó sobre las posibles correlaciones entre las variables AR y años de experiencia profesional.

\section{RESULTADOS}

Como podemos observar en la tabla 1, la mayoría de los bailarines presentan una Ansiedad Rasgo por debajo del centil 40, lo que indica un bajo nivel de AR. 
Los resultados muestran que un 56.8\% de los sujetos presentan una AR situada en el percentil 10 .

La gráfica 1 permite analizar la experiencia profesional de los bailarines estudiados. Si comparamos los resultados de AR del grupo con sujetos más jóvenes, el BBL (11 centiles de media) con los del BON (10) y los del BBL (10) veremos que se puede encontrar una relación entre la edad media de los bailarines y su nivel de AR: los bailarines más jóvenes presentan en general un mayor nivel de AR. Todo ello parece indicar que los bailarines más experimentados presentan niveles de ansiedad diferentes a los más nóveles. Sin embargo, los resultados del análisis de la correlación entre AR y experiencia profesional se revelan no significativos, siendo la media de la variable relativa a la experiencia como profesionales del sector de la danza de los sujetos es 9.34 años.

\begin{tabular}{ccc}
\multicolumn{3}{c}{ Tabla 1. Niveles de AR } \\
\hline Centil (AR) & $\begin{array}{c}\mathrm{N}^{\circ} \text { de sujetos } \\
(n=44)\end{array}$ & $\begin{array}{c}\text { Porcentaje } \\
(n=44)\end{array}$ \\
\hline 10 & 25 & $56.8 \%$ \\
\hline 11 & 4 & $9.1 \%$ \\
\hline 12 & 1 & $2.3 \%$ \\
\hline 15 & 7 & $15.9 \%$ \\
\hline 25 & 6 & $13.6 \%$ \\
\hline 35 & 1 & $2.3 \%$ \\
\hline
\end{tabular}

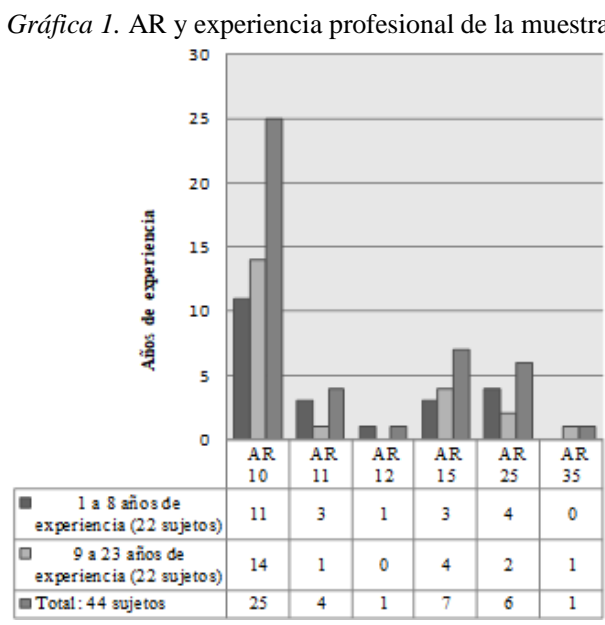

\section{DISCUSIÓN Y CONCLUSIONES}

Por ello, podemos concluir que el bailarín consagrado presenta unos muy bajos niveles de AR que, por otra parte, no se relacionarían de forma significativa con una mayor o menor experiencia en el ámbito profesional. La AR parece ser un rasgo de gran 
estabilidad, o bien realmente característico del bailarín profesional, o no influenciable por el grado de experiencia escénica que éste posea.

Además, podemos concluir que los bailarines profesionales presentan un nivel muy bajo de AR, comparable con el bajo nivel de AR de los deportistas de élite o alto rendimiento.

Se propone una aplicación a la danza de los métodos de entrenamiento psicológico ya utilizados para el deporte de competición, especialmente los relativos al control de la ansiedad y sus nefastas consecuencias.

Es necesario profundizar en esta línea de investigación y estudio, centrándose en los problemas concretos del bailarín y sus posibles tratamientos de reconocimiento, prevención y control, apoyándose sobre lo ya logrado por la Psicología del Deporte.

\section{REFERENCIAS}

De Castro, P. (2011). La ansiedad en el bailarín profesional (Tesis de máster). URJC, Madrid.

Fenz, W.D. (1975). Strategies for coping with stress. En I. Sarason y C. Spielberger (Eds.), Stress and anxiety, 2, (pp. 305-334). Washington: Hemisphere.

Fenz, W.D., y Jones, G.B. (1972). The Effect of Uncertainty on Mastery of Stress: A Case Study. Psychophysiology, 9, 615-619.

Folkins, C., y Sime, W. (1981). Physical fitness training and mental health. American Psychologist, 36, 373-389.

Garfield, S.L. (1987). Ethical issues in research on psychotherapy. Counseling and Values, 31, 115125.

Gould, D., Horn, T., y Spreeman, J. (1983). Sources of stress in junior elite wrestlers. Journal of Sport Psychology, 5, 159-171.

Levitt, E. (1990). The psychology of anxiety. New Jersey: Lawrence Erlbaum Associates Publishers.

Lowe, R. (1973). Stress, arousal and task performance of little league baseball players. Conferencia médica inédita, University of Illinois: Urbana-Champaign.

Martínez-Otero, V. (2003). Teoría y práctica de la educación. Madrid: CCS.

Morgan, W. (1973). Influence of acute physical activity on state anxiety. National College of Physical Education Association for Men Proceedings, 113-121.

Morgan, W.P. (1997). Physical activity and mental health. Washington, D.C.: Taylor and Francis Publishers.

Petruzzello, S.J., Landers, D.M., Hatfield, B.D., Kubitz, K.A., y Salazar, W. (1991). A meta-analysis on the anxiety-reducing effects of acute and chronic exercise. Outcomes and Mechanisms. Sports Medicine, 3, 143-182.

Scanlan, T.K., y Passer, M.W. (1977). The effects of competition trait anxiety and game win-loss on perceived threat in a natural competitive setting. En R.W. Christina y D.M. Landers (Eds.), Psychology of motor behavior and sport-1976 (pp. 157-160). Champaign, Il: Human Kinetics.

Spielberger, C.D. (1966). Theory and research on anxiety. En C.D. Spielberger (Ed.), Anxiety and behavior (pp. 3-20). New York: Academic Press.

Spielberger, C.D. (1983). State-Trait Anxiety Inventory. Palo Alto, CA: Consulting Psychologists Press. 
Spielberger, C.D., y Díaz-Guerrero, R. (1976). Cross-cultural anxiety. Washington, D.C.: Hemisphere/Wiley.

Spielberger, C.D., Gorsuch R.L., y Laushene, R.E. (1971). Manual for the state-trait anxiety inventory. Palo Alto. CA: Consulting Psychologists Press.

Yerkes, R.M., y Dodson, J.D. (1908). The relation of strength of stimulus to rapidity of habitformation. Journal of Comparative Neurology and Psychology, 18, 459-482.

Recibido: 12 de abril de 2019

Recepción Modificaciones: 27 de abril de 2019

Aceptado: 29 de abril de 2019 